\title{
Reactive clusters on a membrane
}

\author{
R Thul and M Falcke \\ Hahn-Meitner Institut, Abteilung Theorie, Glienicker Str. 100, D-14109 Berlin, \\ Germany \\ PACS numbers: 87.16Ac, 05.40.-a
}

\begin{abstract}
We investigate the reaction dynamics of diffusive molecules with immobile binding partners. The fixed reactants build clusters that comprise just a few tens of molecules, which leads to small cluster sizes. These molecules participate in the reaction only if they are activated. The dynamics of activation is mapped to a timedependent size of an active region within the cluster. We focus on the deterministic description of the dynamics of a single cluster. The spatial setup accounts for one of the most important determinants of the dynamics of a cluster, i.e. diffusional transport of reaction partners toward or away from the active region of the cluster. We provide numerical and analytical evidence that diffusion influences decisively the dynamic regimes of the reactions. The application of our methods to intracellular $\mathrm{Ca}^{2+}$ dynamics shows that large local concentrations saturate the $\mathrm{Ca}^{2+}$ feedback to the channel state control. That eliminates oscillations depending on this feedback.
\end{abstract}

\section{Introduction}

A basic task of cells is to respond to inner and outer stimuli, which involves sequences of chemical reactions that form the signaling pathway. While numerous reactions take place between dissolved binding partners in the cytosol, others occur in the plasma or organelle membranes. A third class of reactions happens between cytosolic molecules and membrane-bound reaction partners. Often, these reactions involve only a small region of the membrane at a time because the cell does not only interpret the total amount of additional substances, but also the precise location of its production. A wellknown example of this type of reaction is in the formation of the second messenger cyclic adenosine monophosphate (cAMP). Dissolved adenosine triphosphate (ATP) binds to adenylyl cylcase, which is fixed in the plasma membrane, to synthesize cAMP. Depending on where this reaction occurs, it may trigger a break down of glycogen to glycose or gene expression [1].

The fixed membrane-bound reaction partners are often concentrated in a small membrane area, which we call cluster. We will refer to the molecules in the cluster as fixed elements. Generally, not all molecules in the membrane patch participate in the reaction. Just activated elements join in. Although the activating mechanism varies 
among membrane-bound processes, this principle is ubiquitous in biology. A single cluster with several tens of fixed elements is the focus of the present work.

The small number of fixed elements in a cluster entails small cluster areas. This strong localization of the reactions causes large spatial gradients of diffusing species. The cluster diameters are much smaller than the diffusion length of the species in solution. That imposes limits on the reactions due to diffusion of dissolved reaction partners toward or away from the cluster. Here, we will model the dynamics of an active cluster accounting explicitly for the diffusion of dissolved reaction partners.

Beside cAMP, cells employ other second messengers in the above mentioned signaling tasks. $\mathrm{Ca}^{2+}$ is another prominent representative of a second messenger that participates in numerous processes [2]. It communicates the fertilization through an egg cell [3], controls apoptosis [4], and is vital for the excitation-contraction coupling in cardiac myocytes [5]. The mechanism by which a cell regulates the concentration of cytosolic $\mathrm{Ca}^{2+}$ involves receptors on the membrane of intracellular compartments. Here, the inositol-1,4,5 trisphosphate $\left(\mathrm{IP}_{3}\right)$ receptor channel $\left(\mathrm{IP}_{3} \mathrm{R}\right)$ on the membrane of the endoplasmic reticulum (ER) serves as an illustrative example. Upon activation, the channel opens, which in turn results in a transient flux of $\mathrm{Ca}^{2+}$ from the ER to the cytosol. Importantly, the open probability of the channel depends sensitively on the concentration of free cytosolic $\mathrm{Ca}^{2+}$. A moderate increase raises the tendency to release calcium, whereas a high concentration of $\mathrm{Ca}^{2+}$ causes inhibition and closes the channel. Thus, the channel releases the species that controls its state.

The feedback of $\mathrm{Ca}^{2+}$ on the channel dynamics becomes even more relevant when we take the spatial organization of $\mathrm{IP}_{3}$ receptor channels into account. Generically, the receptor channels form clusters that are randomly distributed on the membrane of the ER. The typical inter-cluster distance is $2-7 \mu \mathrm{m}[6]$. The number of $\mathrm{IP}_{3} \mathrm{Rs}$ within a cluster has not yet been established experimentally. However, it is estimated that a cluster comprises 1-40 channels. Using the size of $18 \mathrm{~nm}$ for a single $\mathrm{IP}_{3} \mathrm{R}$ with all four subunits [7], we arrive at a cluster diameter of $18-100 \mathrm{~nm}$. Thus, $\mathrm{IP}_{3}$ receptor channels are tightly coupled by diffusion within a cluster because the $\mathrm{Ca}^{2+}$ concentration decays on length scales of about $1 \mu \mathrm{m}[8]$.

We see that intracellular $\mathrm{Ca}^{2+}$ dynamics is another example of a reaction between partners fixed in a small membrane area and diffusing species. The channels are the fixed elements, $\mathrm{Ca}^{2+}$ is the diffusing species and the reactions are production (here as release) and binding to and dissociation from the binding sites on the channel.

To date, modeling of intracellular $\mathrm{Ca}^{2+}$ dynamics has proceeded along two distinct paths. In the first approach, deterministic models and spatially averaged concentrations have been used. $[9,10]$. Since this approach neglects any spatial information such as gradients, the $\mathrm{Ca}^{2+}$ concentration changes by only one order of magnitude during oscillations. However, simulations show that large gradients occur and that the $\mathrm{Ca}^{2+}$ concentration changes by 3-4 orders of magnitude at an open cluster [8]. Hence, these models do not span the necessary range of $\mathrm{Ca}^{2+}$ concentrations. In the second approach, stochastic methods have been applied taking the small number of fixed elements per 
cluster seriously $[11,12,13,14,15]$. For a review on intracellular calcium models, see [16]. The stochastic simulations have been performed with discrete sources, thereby incorporating gradients. They exhibit oscillations in regions of the parameter space where the deterministic behavior was non-oscillatory. To understand the loss of the oscillatory regime was one motivation of this study. However, our results will apply to many reactions with spatially localized reaction partners which create large gradients of diffusing species. Therefore we present a general formulation of the model in the following section and apply it to intracellular $\mathrm{Ca}^{2+}$ dynamics in the 3rd section.

\section{General Model}

The aim of this section is to introduce a general formulation of a model that incorporates the areally restricted binding dynamics. As motivated in the introduction, we refer to the immobile molecules in the reacting region as fixed elements.

Each element occupies a non-negligible spatial fraction of a cluster because only several tens of closely packed elements reside therein. Therefore, the state of a cluster is well characterized by the area that is occupied by the activated elements. We term this region the active area of a cluster. It usually decomposes into unconnected parts. Nevertheless, we merge the area of all activated elements to one concentric patch. Its size equals the sum of the areas of all activated elements, and its radius is denoted by a. A change in the number of activated elements is translated into an alteration of the radius. If all elements are deactivated, $a$ equals zero. $a$ takes its maximum value $a_{0}$ when all elements are in the activated state. This mapping can be applied if the diffusion length of the diffusing species is larger than the cluster size. The procedure follows results by Swillens and Dupont [17].

We represent the cluster of fixed elements as a sphere with radius $a$. A typical value of the maximal radius $a_{0}$ is tens of nanometers. A second sphere surrounds the cluster concentrically. It has a radius $b=5-100 \mu \mathrm{m}$ and depicts the environment of the cluster.

The system contains $m$ diffusive species . They are described by the concentration fields $c(r, t):=\left\{c_{1}(r, t), \ldots, c_{m}(r, t)\right\}$. Their dynamics are of the general form

$$
\dot{c}=D \nabla_{r}^{2} c+f_{1}(c) \Theta(a-r)+f_{2}(c) \Theta(r-a) .
$$

$\nabla_{r}^{2}$ denotes the radial part of the Laplacian in three dimensions. $\Theta(x)$ with $\Theta(x)=1$ for $x \geq 0, \Theta(x)=0$ otherwise represents the Heaviside step function. The functions $f_{1}$ and $f_{2}$ subsume the details of the dynamics for $r<a$ and $r>a$, respectively. Most commonly, $f_{1}$ is dominated by production and $f_{2}$ by consumption.

The state of a fixed element is controlled by binding of the diffusive species to binding sites. The occupation of these binding sites determines the state. Usually, there are several binding sites per element. We denote with $p_{i}, i=1, \ldots, n$ the fraction of elements in the state $i$ and refer to the $p_{i}$ as gating variables. The dynamics of the gating variables are governed by the general equation

$$
\dot{p}_{i}=g_{i}\left(c, p_{1}, \ldots, p_{n}\right), \quad i=1, \ldots, n .
$$


Some of the $n$ states correspond to the activated state of the elements, so that the fraction of activated elements is determined by the sum of the corresponding gating variables. Consequently, the gating variables determine the radius $a$ of the active area by some function $f$ as

$$
a=f\left(c(a), p_{1}(a), \ldots, p_{n}(a)\right) .
$$

We include a dependence on the diffusive species and on all $n$ gating variables in $f$ to account for the most general case. The values of the concentration fields and of the gating variables do not vary significantly within a cluster because the diffusion lengths are larger than $a_{0}$. Therefore, we can pick a typical value to compute $c$ and $p_{i}$. We have chosen the value at the boundary of the cluster, which turns equation (3) into an implicit expression.

The behavior of the equations (1)-(3) can be investigated by a bifurcation analysis, which determines the stationary states and their stability. We begin with the stationary states, i.e. the solutions of the equations

$$
\begin{aligned}
& 0=D \nabla_{r}^{2} \bar{c}+f_{1}(\bar{c}) \Theta(\bar{a}-r)+f_{2}(\bar{c}) \Theta(r-\bar{a}) \\
& 0=g_{i}\left(\bar{c}, \bar{p}_{1}, \ldots, \bar{p}_{n}\right), \quad i=1, \ldots, n
\end{aligned}
$$

The dash indicates the stationary states of the $\mathrm{Ca}^{2+}$ concentration profile and of the gating variables, respectively. The constant $\bar{a}$ denotes the stationary value of the active area. Equation (4a) can be treated separately for $r<\bar{a}$ and $r>\bar{a}$ due to the Heaviside step function. Since we demand the concentration profiles to be $C^{1}$ functions with respect to $r$, the matching conditions for the stationary solutions read $\bar{c}_{i}(\bar{a})=\bar{c}_{o}(\bar{a})$ and $\partial \bar{c}_{i} / \partial r(\bar{a})=\partial \bar{c}_{o} / \partial r(\bar{a})$. The subscripts $i$ and $o$ indicate the inner and outer solution, respectively. The computation of $\bar{a}$ proceeds in two steps. Firstly, we solve equation (4a) with a fixed, but arbitrary value of $\bar{a}$. That results in a solution for $\bar{c}$, which includes $\bar{a}$ as a still undetermined parameter. Secondly, we determine $\bar{a}$ self-consistently from equation (3) after inserting the solutions for $\bar{c}$ and for $\bar{p}_{i}$.

Figure 2 shows a graphical method to determine $\bar{a}$. The dotted line is the bisection line, whereas the full lines represent the rhs of equation (3) for a specific model (see below). The stationary states are given by the intersections. Upon changing one parameter, the curve of $f$ is shifted. It results in a change of the values or the number of fixed points. The existence of a saddle node bifurcation is easily deduced from such a plot. It occurs when $f$ touches the bisection line. That is equivalent to the condition $f^{\prime}(a)=1$.

Knowing the stationary points $(\bar{c}, \bar{a})$, we investigate their stability. A linearization of the reaction-diffusion dynamics in (1) and the gating dynamics in (2) results in the 
equations

$$
\begin{aligned}
\dot{y}= & D \nabla_{r}^{2} y+\left\{f_{1}(\bar{c})-f_{2}(\bar{c})\right\} \delta_{D}(\bar{a}-r) \delta a(y, z) \\
& +\left\{\frac{\partial f_{1 i}}{\partial c_{j}}(\bar{c}) \Theta(\bar{a}-r)+\frac{\partial f_{2 i}}{\partial c_{j}}(\bar{c}) \Theta(r-\bar{a})\right\} \cdot y \\
\dot{z}_{i}= & \sum_{j=0}^{m} \frac{\partial g_{i}}{\partial c_{j}}\left(\bar{c}, \bar{p}_{1}, \ldots, \bar{p}_{n}\right) y_{j}+\sum_{j=0}^{n} \frac{\partial g_{i}}{\partial p_{j}}\left(\bar{c}, \bar{p}_{1}, \ldots, \bar{p}_{n}\right) z_{j}
\end{aligned}
$$

We define $y$ as the perturbations $\delta c$ of the diffusing species, and $z_{i}$ as the perturbations $\delta p_{i}$ of the gating variables. $\delta_{D}$ denotes Dirac's delta function. Although $a$ is not a dynamical variable in our model, it still changes in time. That is a consequence of equation (3) because $a$ is computed from the evolving concentration fields and gating variables. $a$ can be written as $a=\bar{a}+\delta a$ with $\delta a=\delta a(y, z)$. To evaluate $\delta a$ from equation (3), we expand the expression

$$
\bar{a}+\delta a=\left.f(\bar{c}(r)+y(r), \bar{p}(r)+z(r))\right|_{r=\bar{a}+\delta a}
$$

to linear order:

$$
\delta a=\frac{\sum_{i=1}^{m} \frac{\partial f}{\partial c_{i}} y(\bar{a})+\sum_{i=1}^{n} \frac{\partial f}{\partial p_{i}} z_{i}(\bar{a})}{1-\sum_{i=1}^{m} \frac{\partial f}{\partial c_{i}} \frac{\partial \bar{c}_{i}}{\partial r}(\bar{a})-\sum_{i=1}^{n} \frac{\partial f}{\partial p_{i}} \frac{\partial \bar{p}_{i}}{\partial r}(\bar{a})} .
$$

The derivatives of $f$ have to be taken at $(\bar{c}(\bar{a}), \bar{p}(\bar{a}))$. The denominator only arises because of the evaluation of $f$ at $r=a$ in equation (3).

When we combine $y$ and $z$ to an $n+m$ dimensional vector $x=(y, z)^{t}$, the linearized equations take the matrix form $\dot{x}=M x$. If $M$ can be diagonalized, the general solution for $x$ is given by a linear combination of terms $v_{i} \exp \left(\omega_{i}\right) . v_{i}$ represents an eigenvector of $M$ and $\omega_{i}$ the corresponding eigenvalue. Consequently, the linear stability is uniquely determined by the eigenvalues of $M$. As shown in the appendix, $M$ can be diagonalized. The eigenvalues $\lambda_{i}$ that originate from the gating variables constitute a subset $\left\{\lambda_{i}\right\}$ of all eigenvalues $\left\{\omega_{i}\right\}$ and are all non-positive, i.e. $\lambda_{i} \leq 0 \forall i$, if the gating variable dynamics are rate equations derived from a master equation. Moreover, the eigenvectors $v_{i}$ of $M$ that correspond to the eigenvalues $\lambda_{i}$ possess the structure $v_{i}=\left(0, \ldots, 0, q_{i}\right)^{t}$ with $\operatorname{dim} q_{i}=n$. That has two important consequences. Firstly, the eigenvalues from the gating dynamics do not contribute to any linear instability. Secondly, the solution for $y$ does not depend on $\exp \left(\lambda_{i} t\right)$. Therefore, the time dependence of $y$ is solely governed by the eigenvalues that originate from equations (8) and $(5 \mathrm{a})$.

Equation (5a) can be solved separately for $r<\bar{a}$ and $r>\bar{a}$ because of the Heaviside step function. The matching conditions are now $y_{i}(\bar{a})=y_{o}(\bar{a})$, and the first derivative jumps according to

$$
\left[\frac{d y_{o}}{d r}-\frac{d y_{i}}{d r}+\frac{f_{1}(\bar{c})-f_{2}(\bar{c})}{D} \delta a(y, z)\right]_{\bar{a}}=0
$$


due to Dirac's delta function in equation (5a). The continuity at $\bar{a}$ and equation (8) fix the still undetermined coefficients of $y$. The resulting system of equations is homogeneous. It has a non-trivial solution only if the determinant vanishes. That yields an implicit equation for the eigenvalues of the concentration fields and thus determines their linear stability.

\section{Calcium dynamics}

We now apply the method of section 2 to the dynamics of cytosolic calcium as a prototypic model system. The cytosolic $\mathrm{Ca}^{2+}$ concentration $c$ is governed by

$$
\dot{c}=D \nabla^{2} c+k_{l}(E-c)-k_{p} c+k_{c}(E-c) \Theta(a-r) .
$$

The constants $D$ and $E$ denote the diffusion coefficient of $\mathrm{Ca}^{2+}$ in the cytosol and the $\mathrm{Ca}^{2+}$ concentration in the ER, respectively. The term $k_{l}(E-c)$ refers to a leak current, whereas $k_{p} c$ describes the calcium uptake by the ER through sarco-endoplasmic reticulum calcium ATPase (SERCA) pumps. Although it would be more realistic to model SERCAs by a Hill equation with coefficient 2, we approximate them by a linear expression for the sake of an analytical treatment. The last term in equation (9) describes the flux of $\mathrm{Ca}^{2+}$ through $\mathrm{IP}_{3}$ receptor channels. They represent the fixed elements, and the radius $a$ of the active area is determined by the fraction of open $\mathrm{IP}_{3}$ receptor channels.

The state of a single $\mathrm{IP}_{3} \mathrm{R}$ is controlled by the state of its four subunits [18]. Each subunit expresses binding sites for $\mathrm{Ca}^{2+}$ and $\mathrm{IP}_{3}$. Their occupation determines the state of the subunit. De Young and Keizer introduced a model to describe the dynamics of one subunit [9]. It consists of three binding sites: an activating $\mathrm{Ca}^{2+}$ binding site, an inhibitory $\mathrm{Ca}^{2+}$ binding site and an $\mathrm{IP}_{3}$ binding site. Therefore, the state of a subunit can be specified by a binary triplet $i j k$. The first index represents the $\mathrm{IP}_{3}$ binding site, the second the activating $\mathrm{Ca}^{2+}$ binding site and the last the inhibiting $\mathrm{Ca}^{2+}$ binding site. An index equals 1 when a site is occupied and 0 otherwise. The 8 states that originate from the three binding sites are depicted in figure 1. Each state corresponds to one corner of the cube. The transition rates between the different states are indicated at the arrows. Binding of $\mathrm{Ca}^{2+}$ and $\mathrm{IP}_{3}$ is always proportional to the $\mathrm{Ca}^{2+}$ and $\mathrm{IP}_{3}$ concentration, respectively, whereas unbinding is independent from these concentrations.

Since a subunit is activated when $\mathrm{IP}_{3}$ and activating $\mathrm{Ca}^{2+}$ are bound, $p_{110}$ denotes the fraction of open subunits. The probability to find a conducting $\mathrm{IP}_{3} \mathrm{R}$ is $4 p_{110}^{3}-3 p_{110}^{4}$ because a channel is open when at least three out of four subunits are activated. The size of the active area is set to a fraction of its maximal value corresponding to the above probability, so that

$$
a=a_{0} p_{110} \sqrt[3]{4-3 p_{110}} .
$$




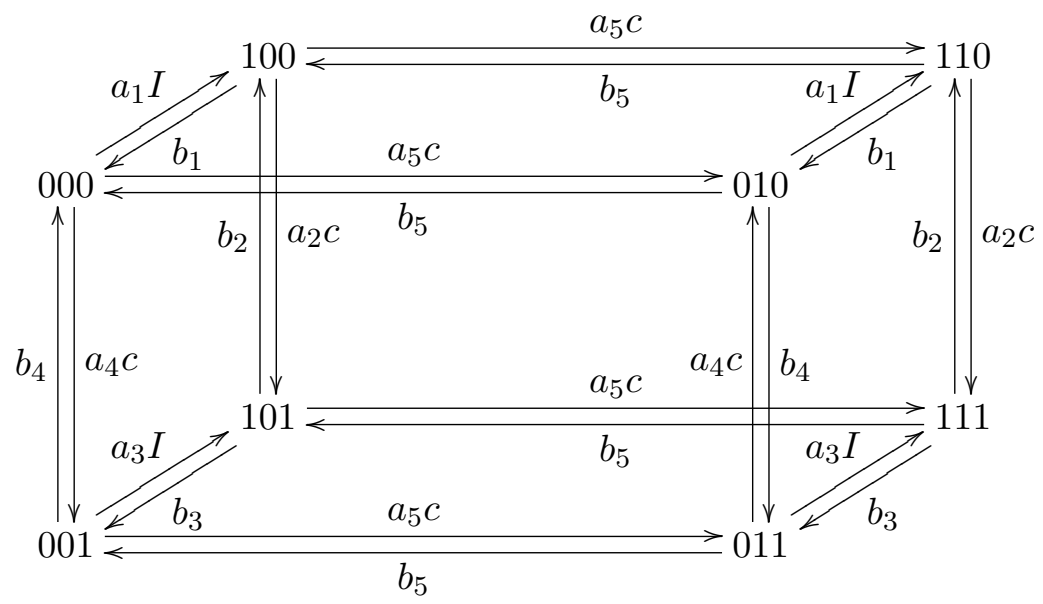

Figure 1. Transition scheme of the De Young Keizer model. The dissociation constants $d_{i}$ are defined as $d_{i}:=b_{i} / a_{i}$.

That is the specification of the function $\mathrm{f}$ (see equation (3)) for the $\mathrm{Ca}^{2+}$ dynamics of the De Young Keizer (DK) model. Our investigations of the $\mathrm{Ca}^{2+}$ dynamics start with the stationary solution of (9) for an arbitrary value of $\bar{a}$ :

$$
\begin{aligned}
\bar{c}(r) & =\left\{\bar{B}(\bar{a}) \frac{\exp \left(-\bar{k}_{2} r\right)}{r}+\frac{k_{l} E}{k_{l}+k_{p}}\right\} \Theta(r-\bar{a}) \\
& +\left\{\bar{A}(\bar{a}) \frac{\sinh \left(\bar{k}_{1} r\right)}{r}+\frac{\left(k_{l}+k_{c}\right) E}{k_{l}+k_{p}+k_{c}}\right\} \Theta(\bar{a}-r)
\end{aligned}
$$

with

$$
\begin{aligned}
\bar{A}(a) & =\frac{k\left(\bar{k}_{2} a+1\right)}{\cosh \left(\bar{k}_{1} a\right) \bar{k}_{1}+\sinh \left(\bar{k}_{1} a\right) \bar{k}_{2}}, \\
\bar{B}(a) & =\frac{k\left(\sinh \left(\bar{k}_{1} a\right)-\cosh \left(\bar{k}_{1} a\right) \bar{k}_{1} a\right)}{\exp \left(-\bar{k}_{2} a\right)\left(\cosh \left(\bar{k}_{1} a\right) \bar{k}_{1}+\sinh \left(\bar{k}_{1} a\right) \bar{k}_{2}\right)}, \\
k & =\frac{-k_{c} k_{p} E}{\left(k_{l}+k_{p}+k_{c}\right)\left(k_{l}+k_{p}\right)}
\end{aligned}
$$

and

$$
\bar{k}_{1}=\sqrt{\frac{k_{l}+k_{p}+k_{c}}{D}}, \quad \bar{k}_{2}=\sqrt{\frac{k_{l}+k_{p}}{D}} .
$$

We applied $\partial \bar{c} / \partial r(0)=0$ and $c(b)=k_{l} E /\left(k_{l}+k_{p}\right)$ as boundary conditions. The latter complies with the base level of the system. The stationary value of $p_{110}$ in dependence on $\bar{c}$ and the $\mathrm{IP}_{3}$ concentration $I$ reads

$$
\bar{p}_{110}=\frac{d_{2} I}{\left(\bar{c}+d_{5}\right)\left(d_{1} d_{2}+\bar{c} d_{3}+\bar{c} I+d_{2} I\right)} .
$$

Here, $d_{1}$ and $d_{3}$ denote the dissociation constants for $\mathrm{IP}_{3}$ when no $\mathrm{Ca}^{2+}$ and when $\mathrm{Ca}^{2+}$ is bound to the inhibiting site, respectively. The parameters $d_{2}$ and $d_{4}$ refer to the 
dissociation constants for the inhibiting $\mathrm{Ca}^{2+}$ processes dependent on $\mathrm{IP}_{3}$ binding. $d_{5}$ represents the dissociation constant for the activating $\mathrm{Ca}^{2+}$ site [9].

Inserting $\bar{p}_{110}$ with $\bar{c}=\bar{c}(a)$ into equation (10) determines the stationary values of $a$. They correspond to the intersections of the dotted bisection line and the curve of $f$ depicted as solid lines in figure 2 . When we increase the $\mathrm{IP}_{3}$ concentration $I$, the curve of $f$ is shifted upwards. Although $\partial f / \partial I \geq 0$ always holds, the effect on the number of stationary points depends on the parameter values. There is one fixed point at low $I$ for the parameter values chosen in figure 2 . Three stationary values exist at an intermediate regime, and one stationary point is present at high $\mathrm{IP}_{3}$ concentrations. Thus, two saddle node bifurcations occur upon increasing $I$. For other parameter values, we find only one fixed point for the whole range of the $\mathrm{IP}_{3}$ concentration or just a single saddle node bifurcation (see below).

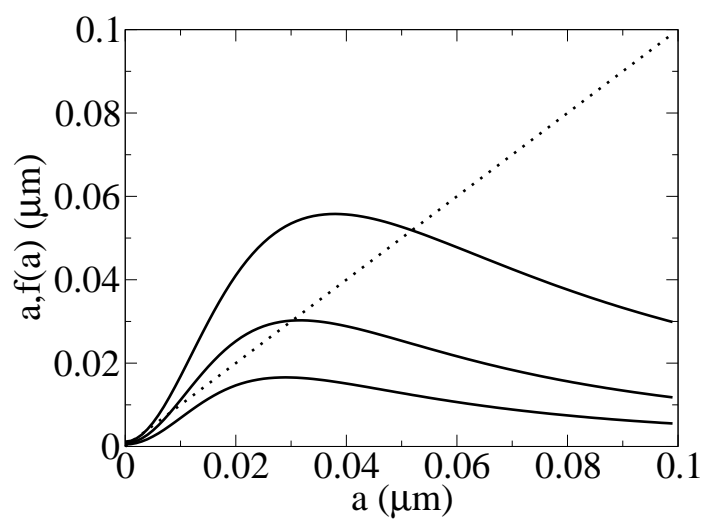

Figure 2. Stationary values of $a$ given by the intersections of the bisection line (dotted) and the curve of $f$ (solid). For $f, I$ increases from bottom to top. Parameters values are $d_{1}=0.13 \mu \mathrm{M}, d_{2}=3 \mu \mathrm{M}, d_{3}=0.9434 \mu \mathrm{M}, d_{4}=0.4133 \mu \mathrm{M}, d_{5}=0.8234 \mu \mathrm{M}, k_{p}=$ $80 \mathrm{~s}^{-1}, k_{l}=0.002 \mathrm{~s}^{-1}, k_{c}=34500 \mathrm{~s}^{-1}, E=750 \mu \mathrm{M}, a_{0}=0.03 \mu \mathrm{m}, D=40 \mu \mathrm{m}^{2} \mathrm{~s}^{-1}$

The DK model assumes that the $\mathrm{IP}_{3}$ dynamics is much faster than calcium binding and unbinding. That entails a fast equilibration between states with $\mathrm{IP}_{3}$ bound and not bound. We eliminate the $\mathrm{IP}_{3}$ dynamics adiabatically in the following and use the stationary values of the states with $\mathrm{IP}_{3}$ bound and not bound. As shown in the appendix, the value of $\bar{p}_{110}$ is not changed by this approximation. Thus, the above analysis remains valid and we proceed to the stability of the fixed points.

The linearization of equation (9) results in

$$
\dot{y}=D \nabla_{r}^{2} y-\left(k_{l}+k_{p}\right) y-\Theta(\bar{a}-r) k_{c} y+f_{c} \delta_{D}(r-\bar{a}) .
$$

We define $f_{c}:=k_{c}(E-\bar{c}) \delta a$. Note that the inner concentration field $y_{i}$ is still restricted to $r \leq \bar{a}$. In linear order, the varying value of $a$ is translated into an additional flux density $f_{c}$ at the rim of the stationary active area. The solution of $(14)$ is $y(r, t)=\exp (\omega t) u(r)$ with

$$
u(r)=A \frac{\sinh \left(k_{1} r\right)}{r} \Theta(\bar{a}-r)+B \frac{\exp \left(-k_{2} r\right)}{r} \Theta(r-\bar{a})
$$


and

$$
k_{1}=\sqrt{\frac{k_{l}+k_{p}+k_{c}+\omega}{D}}, \quad k_{2}=\sqrt{\frac{k_{l}+k_{p}+\omega}{D}} .
$$

We used the boundary conditions $\partial u / \partial r(0)=0$ and $u(b)=0$. The still unknown coefficients $A$ and $B$ are fixed by the continuity of $u$ and the discontinuity of $\partial u / \partial r$ at $\bar{a}$ (see also equation (8)). The latter is a direct consequence of the last term in equation (14). The homogeneous system of equations for $A$ and $B$ possesses a non-trivial solution only if its determinant equals zero. This leads to the equation

$$
k_{2}+k_{1} \operatorname{coth}\left(k_{1} \bar{a}\right)-\frac{k_{c}(E-\bar{c}(\bar{a}))}{D} \eta=0
$$

that determines the eigenvalue $\omega . \eta$ is given by $\bar{a} \delta a / \sinh \left(k_{1} \bar{a}\right)$, which can be cast into the form

$$
\eta=\frac{\kappa(\omega)}{1-\kappa(0) \frac{\partial \bar{c}}{\partial r}(\bar{a})}
$$

with

$$
\kappa(\omega)=\left[a_{0} \frac{4\left(1-\bar{p}_{110}\right)}{\sqrt[3]{\left(4-3 \bar{p}_{110}\right)^{2}}} \frac{\bar{p}_{110}}{\bar{c}}\left\{\frac{d_{5}}{\frac{\omega}{a_{5}}+d_{5}+\bar{c}}-\frac{\bar{c}}{\frac{\omega}{a_{6}}+d_{6}+\bar{c}}\right\}\right]_{\bar{a}}
$$

and $a_{6}=\left(a_{2} I+d_{1} a_{4}\right) /\left(I+d_{1}\right)$. If the system exhibits a zero eigenvalue bifurcation for a given pair $(\bar{a}, I)$, then $\omega=0$ should solve equation (17). Indeed, using the identity

$$
\frac{k_{c}}{D} \frac{E-\bar{c}(\bar{a})}{\bar{k}_{2}+\bar{k}_{1} \operatorname{coth}\left(\bar{k}_{1} \bar{a}\right)}=\frac{\partial \bar{A}}{\partial a}(\bar{a}) \frac{\sinh \left(\bar{k}_{1} \bar{a}\right)}{\bar{a}},
$$

equation (17) can be transformed to $1=f^{\prime}(\bar{a})$. That is one of the conditions for a saddle node bifurcation.

\section{Results and discussion}

Some results obtained with the model concerning $\mathrm{Ca}^{2+}$ dynamics have already been reported in [19]. Here, we consider parameter values beyond the range relevant for intracellular $\mathrm{Ca}^{2+}$ release by $\mathrm{IP}_{3} \mathrm{Rs}$ to demonstrate model behavior which might be significant for other membrane-bound reactions.

Diffusion of calcium plays a central role for the selection of dynamic regimes of the $\mathrm{Ca}^{2+}$ dynamics besides the dynamics of the $\mathrm{IP}_{3}$ receptor channel. Hence, we present results for $D=40 \mu \mathrm{m}^{2} \mathrm{~s}^{-1}$ and $D=220 \mu \mathrm{m}^{2} \mathrm{~s}^{-1}$. Diffusion of $\mathrm{Ca}^{2+}$ can be easily changed in experiments by application of $\mathrm{Ca}^{2+}$-binding proteins (buffers).

The original DK model is based on a continuous distribution of $\mathrm{IP}_{3}$ receptor channels. Two Hopf bifurcations bounding an oscillatory regime are the most prominent

features of the $\mathrm{Ca}^{2+}$ dynamics. We test whether this property is conserved when going from spatially continuous source terms in equation (9) to a discrete model. To this aim, we rescale the flux density with a typical cluster spacing $R$ and a representative cluster 
radius $a_{0}$ while keeping the total flux constant, i.e. $k_{c}=k_{c}^{D K} R^{3} / a_{0}^{3}$. The resulting $k_{c}$ of $310^{5} \mathrm{~s}^{-1}$, which agrees well with realistic values [8], leads to a loss of the oscillatory regime. We find a single stationary state for all $\mathrm{IP}_{3}$ concentrations, which is linearly stable. Decreasing the flux density by several orders of magnitude and thus approaching the $\mathrm{Ca}^{2+}$ concentration values of the DK model does not restore oscillations. That holds because gradients still prevail.

These results do not mean that a model like equations (1)-(3) does not exhibit oscillations for some parameter values. In the following, we investigate oscillations of the model. In order to obtain oscillations, we choose parameter values supported by experiments, but a value of the activating $\mathrm{Ca}^{2+}$ dissociation constant $d_{5}$ such that we obtain an oscillatory regime. We use $d_{2}=3 \mu \mathrm{M}$ for the inhibitory process in agreement with recent measurements [20] (Mak et. al found dissociation constants up to $45 \mu \mathrm{M}$, but based on a specific model [21]). According to the experiments in [20], the coefficients for binding to the inhibiting site, $a_{2}$ and $a_{4}$, are both set to $0.2(\mu \mathrm{Ms})^{-1}$. The binding rate constant to the activating site can be evaluated from puff frequencies [22]. That implies $a_{5} \geq 1(\mu \mathrm{Ms})^{-1}$. Assays of the dissociation constant for $\mathrm{Ca}^{2+}$ activation yield values from $77 \mathrm{nM}$ to $309 \mathrm{nM}[23,24,25]$. We chose $d_{5}=0.823 \mu \mathrm{M}$, which is motivated by the results depicted in figure 3 . It shows the dynamic regimes of the model in dependence on $d_{5}$ and $I$. Oscillations occur only for larger values of $d_{5}$.

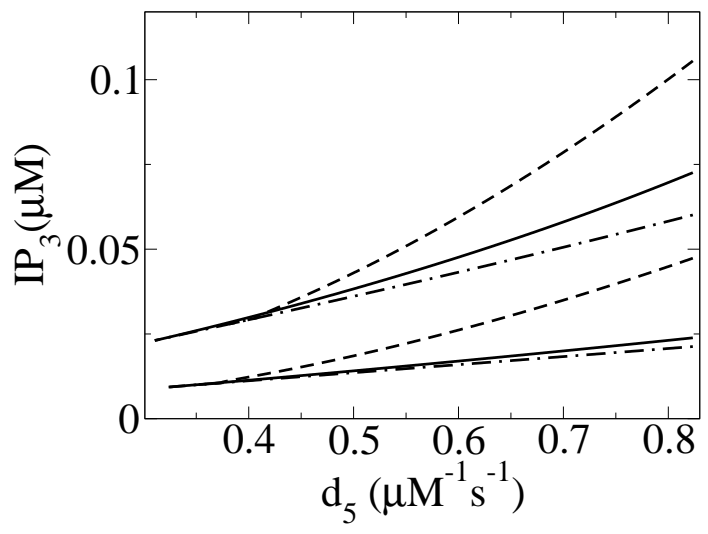

Figure 3. $\mathrm{IP}_{3}$ concentration of the saddle node bifurcations (solid and chain dotted lines) and the Hopf bifurcation (dashed line) in dependence on $d_{5}$. Upper group $D=220 \mu \mathrm{m}^{2} \mathrm{~s}^{-1}$, lower group $D=40 \mu \mathrm{m}^{2} \mathrm{~s}^{-1}$. Parameter values are $d_{1}=0.13 \mu \mathrm{M}, d_{2}=$ $3 \mu \mathrm{M}, d_{3}=0.9434 \mu \mathrm{M}, d_{4}=0.4133 \mu \mathrm{M}, k_{p}=80 \mathrm{~s}^{-1}, k_{l}=0.002 \mathrm{~s}^{-1}, k_{c}=34500 \mathrm{~s}^{-1}, E=$ $750 \mu \mathrm{M}, a_{0}=0.03 \mu \mathrm{m}, a_{2}=a_{4}=0.2(\mu \mathrm{Ms})^{-1}, a_{5}=1(\mu \mathrm{Ms})^{-1}$.

Two saddle node bifurcations and a Hopf bifurcation terminate in a cusp. The oscillations arising at the Hopf bifurcation vanish via a bifurcation close to the lower saddle node bifurcation, which involves an increase in period. We assume it to be a homoclinic bifurcation. Typical oscillations are shown in figure 4 and in ref. [19] for smaller values of $a_{5}$. The pattern is the same for all examples. Upon increasing the $\mathrm{IP}_{3}$ concentration, the system responds with a huge spike of $\mathrm{Ca}^{2+}$ release and finally settles 

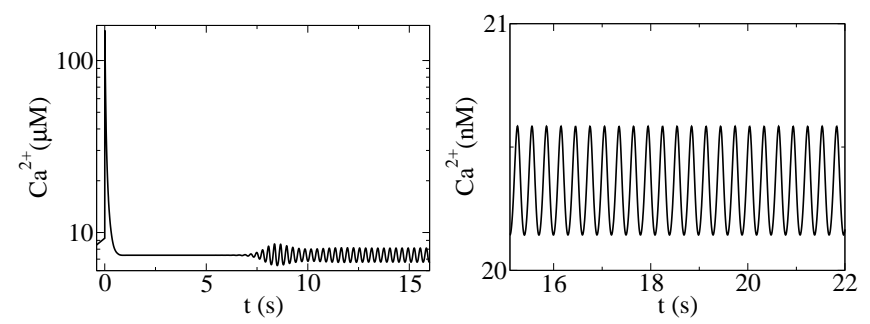

Figure 4. Oscillations of the $\mathrm{Ca}^{2+}$ concentration at $r=0 \mu \mathrm{m}$ (left) and $r=1.588 \mu \mathrm{m}$ (right). Note the difference in the order of magnitude for the amplitude. Parameter values as in Fig 3 and $D=40 \mu \mathrm{m}^{2} \mathrm{~s}^{-1}, a_{5}=10(\mu \mathrm{Ms})^{-1}$.

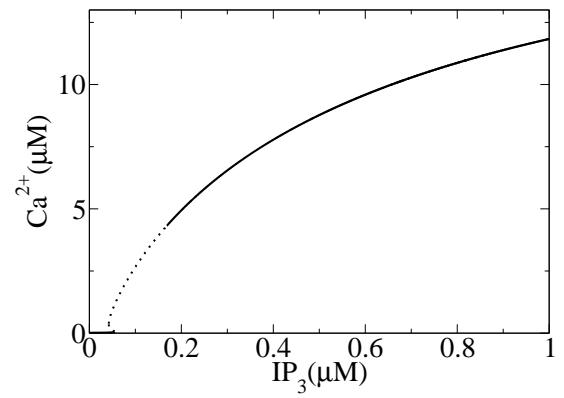

Figure 5. Stationary values of the $\mathrm{Ca}^{2+}$ concentration for $D=40 \mu \mathrm{m}^{2} \mathrm{~s}^{-1}$. Solid lines denote linearly stable fixed points, dotted lines linearly unstable fixed points. Parameter values as in Fig 3 and $d_{5}=1.6468 \mu \mathrm{M}$.

into small amplitude oscillations in the range of the inhibiting dissociation constants. The amplitude decays in space to negligible values within $1.6 \mu \mathrm{m}$ (figure 4 , right panel). Such oscillations had to be expected, since a feedback of $\mathrm{Ca}^{2+}$ to a control process is only given in a range where the process is sensitive to changes of $c$. This range is around the dissociation constant $d_{5}$ for $\mathrm{Ca}^{2+}$ activation and $d_{2}$ and $d_{4}$ for $\mathrm{Ca}^{2+}$ inhibition. Changes of concentrations far above or below the dissociation constants do not exert a feedback on the dynamics [26]. Hence, the large difference between dissociation constants and concentration values occurring at the releasing channel lead to saturation of the control mechanisms of the $\mathrm{IP}_{3} \mathrm{R}$ by $\mathrm{Ca}^{2+}$. That is the reason for the small oscillatory regimes and amplitudes.

Figure 5 shows that the structure of the bifurcation diagram does not change with a higher value of the activating dissociation constant. There is a single fixed point for almost all $\mathrm{IP}_{3}$ concentrations. Stable limit cycles exist close to the bistable area. They only extend to $\mathrm{IP}_{3}$ concentrations where the upper branch is unstable. Thus, we again find a very small band of $\mathrm{IP}_{3}$ concentrations in which the system oscillates. The oscillations behave in the same way as described above.

The existence of oscillations does not solely depend on dissociation constants. It depends on rate constants as well. Therefore, we test the influence of the binding rate constant $a_{5}$ for $\mathrm{Ca}^{2+}$ activation and of the binding rate constants $a_{2}$ and $a_{4}$ for $\mathrm{Ca}^{2+}$ 
inhibition on the stationary states. The concentration values of the stationary states are conserved, since we do not change the dissociation constants.

The Hopf bifurcation moves toward the left saddle node bifurcation when we increase the rates for the inhibitory processes. Figure 6 displays the difference between the $\mathrm{IP}_{3}$ concentration values of the Hopf bifurcation and those of the left saddle node bifurcation. This difference decreases monotonically to zero with higher values of $a_{2}$. Hence, the oscillatory regime shrinks for stronger inhibition. The opposite effect occurs for $\mathrm{Ca}^{2+}$ activation. An increment of $a_{5}$ (while keeping $d_{5}$ constant) shifts the Hopf bifurcation to higher $\mathrm{IP}_{3}$ concentrations.

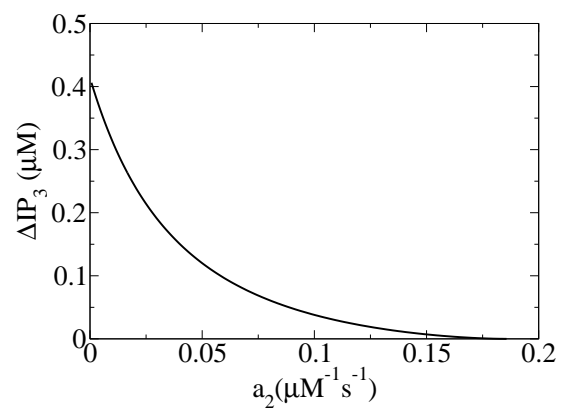

Figure 6. Difference between the $\mathrm{IP}_{3}$ concentration of the Hopf bifurcation and the left saddle node bifurcation in dependence on $a_{2}$. Parameter values as in Fig. 8 and $D=40 \mu \mathrm{m}^{2} \mathrm{~s}^{-1}, a_{2}=a_{4}$.

Oscillations do not always disappear in a (putative) homoclinic bifurcation. The results in figure 7 illustrate a period doubling sequence while approaching the lower saddle node bifurcation. The left panel shows a period-2 example; the right panel depicts a period-4 example. Higher periods occur, too. Oscillations appear only when the upper branch is unstable for these parameter values, too, which leads to a small oscillatory regime. The oscillations are again considerably damped at a distance of $1.6 \mu \mathrm{m}$.
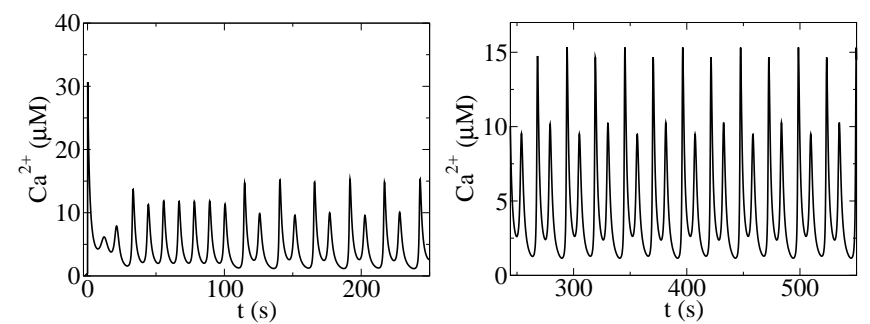

Figure 7. Oscillations of the $\mathrm{Ca}^{2+}$ concentration at $r=0 \mu \mathrm{m}$ for different values of the $\mathrm{IP}_{3}$ concentration. At $t=100 \mathrm{~s}$, we decreased $I$ from $0.22 \mu \mathrm{M}$ to $0.218 \mu \mathrm{M}$ (left panel), whereas $I=0.215 \mu \mathrm{M}$ for all times in the right panel. Parameter values as in Fig 8 and $D=50 \mu \mathrm{m}^{2} \mathrm{~s}^{-1}$.

We find a different structure of the bifurcation diagram for parameter values like those in figure 8. For $D=40 \mu \mathrm{m}^{2} \mathrm{~s}^{-1}$, there are two saddle node bifurcations and a 

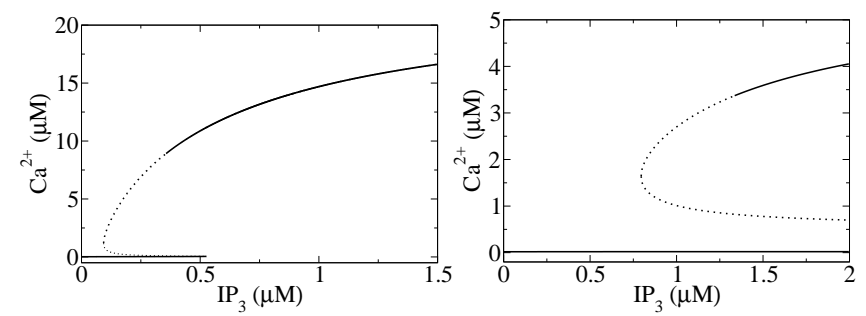

Figure 8. Stationary values of the $\mathrm{Ca}^{2+}$ concentration for $D=40 \mu \mathrm{m}^{2} \mathrm{~s}^{-1}$ (left) and $D=220 \mu \mathrm{m}^{2} \mathrm{~s}^{-1}$ (right). Solid lines denote linearly stable fixed points, dotted linearly unstable fixed points. Parameter values are $d_{1}=0.13 \mu \mathrm{M}, d_{2}=12.588 \mu \mathrm{M}, d_{3}=$ $0.9434 \mu \mathrm{M}, d_{4}=1.7346 \mu \mathrm{M}, d_{5}=2.4702 \mu \mathrm{M}, k_{p}=80 \mathrm{~s}^{-1}, k_{l}=0.002 \mathrm{~s}^{-1}, k_{c}=$ $700 \mathrm{~s}^{-1}, E=750 \mu \mathrm{M}, a_{0}=0.11 \mu \mathrm{m}, a_{2}=a_{4}=0.0167(\mu \mathrm{M} s)^{-1}, a_{5}=0.667(\mu \mathrm{Ms})^{-1}$.

Hopf bifurcation. That is similar to the results obtained above. However, increasing the diffusion coefficient changes the topology of the bifurcation diagram. A value of $D=220 \mu \mathrm{m}^{2} \mathrm{~s}^{-1}$ yields only one saddle node bifurcation. It leads to a bistable regime that extends infinitely toward high $\mathrm{IP}_{3}$ concentration values.

These two examples illustrate that diffusion influences essentially the dynamical behavior. Generally, the impact of diffusion on the fixed points can be deduced from $\partial \bar{c} / \partial D$. The derivative simplifies to

$$
\frac{1}{\zeta} \frac{\partial \bar{c}}{\partial D}(a)=\frac{\partial \bar{k}_{1}}{\partial D}\left(\bar{k}_{2} a+1\right)\left\{\bar{k}_{1}-\frac{\sinh \left(2 \bar{k}_{1} a\right)}{2 a}\right\}+\frac{\partial \bar{k}_{2}}{\partial D}\left\{\frac{\sinh \left(2 \bar{k}_{1} a\right)}{2} \bar{k}_{1}-\frac{\sin ^{2}\left(\bar{k}_{1} a\right)}{a}\right\}
$$

with

$$
\zeta=\frac{k}{\left(\cosh \left(\bar{k}_{1} a\right) \bar{k}_{1}+\sinh \left(\bar{k}_{1} a\right) \bar{k}_{2}\right)^{2}} .
$$

Since $\partial \bar{k}_{i} / \partial D=-\bar{k}_{i} /(2 D), i=1,2$, we immediately arrive at $\partial \bar{c} / \partial D(a)<0$, i.e. $c(a)$ decreases when $D$ is increased. The dependence of $c(a)$ on $a$ is a monotonously increasing one with increasing $a$. Hence, if we increase $a$, we can increase $D$ to such an extent that $c(a)$ does not change. That entails that for any $\Delta>0$, there exists an $\alpha>0$ so that $f_{D+\Delta}(a+\alpha)=f_{D}(a)$. The statement implies a stretching of $f$ to the right upon increasing the diffusion coefficient. The exact effect on the stationary points depends on their properties. A continuous increment of $D$ leads first to a higher value of $\bar{a}$ and then to the disappearance of the fixed point, when $\partial f(\bar{a}) / \partial a<0$. In the case of figure $8, f$ is stretched to such an extent that an increment of $I$ does not shift $f$ upwards enough to cause a second saddle node bifurcation (see figure 2).

\section{Conclusion and outlook}

We have presented an extended study of a new modeling concept for diffusive species that react with immobile reaction partners. The fixed reactants are confined to small clusters. Our approach to describe the cluster dynamics is always applicable when the diffusion length is much larger than the cluster size. We applied the above method to 
the dynamics of intracellular calcium mediated by $\mathrm{IP}_{3}$ receptor channels. The spatial restriction of the $\mathrm{Ca}^{2+}$ flux to small membrane areas led to the disappearance of $\mathrm{Ca}^{2+}$ oscillations computed in spatially continuous models. The enlarged values of the $\mathrm{Ca}^{2+}$ concentration at the cluster resulted in a single linearly stable stationary state. Choosing smaller values of the channel flux constant $k_{c}$ did not restore $\mathrm{Ca}^{2+}$ oscillations. Hence, the strong impact of spatial gradients on dynamic regimes will most likely apply as well to localized reactions generating much smaller gradients than the gradients around a releasing $\mathrm{Ca}^{2+}$ channel.

The flux constants we have used and which entail the large concentrations occurring at releasing clusters are based on recent simulation results. These simulations of $\mathrm{Ca}^{2+}$ liberation close to experimental conditions show that $\mathrm{Ca}^{2+}$ concentrations span the range of $25-170 \mu \mathrm{M}$ in the center of an open cluster [8]. That is $3-4$ orders of magnitude larger than the base level. At the same time, the concentration increases only 1-2 times base level at neighboring clusters. The existence of propagating waves proofs that the activation process is sensitive to these small concentration changes. Since the channel control processes experience concentration changes of several orders of magnitude and react to small changes already, the possibility to eliminate large concentrations from the dynamics by rescaling of binding constants must be ruled out.

Even if the $\mathrm{Ca}^{2+}$ concentration oscillated according to a deterministic model, these oscillations are not the oscillations observed in experiments [19]. Two observations led to this conclusion. Firstly, the range of $\mathrm{IP}_{3}$ concentrations providing oscillations is too small. Secondly, the amplitude as well as the mean of the oscillations are already considerably damped in a distance of $1.6 \mu \mathrm{m}$ from the cluster. Thus, they cannot represent the global $\mathrm{Ca}^{2+}$ oscillations seen in experiments. The spatial damping of the oscillations applies to each reaction that produces a diffusing species.

Our findings support earlier results that deterministic models, including only activation by $\mathrm{IP}_{3}$, activation by $\mathrm{Ca}^{2+}$ and inhibition by $\mathrm{Ca}^{2+}$, do not capture intracellular $\mathrm{Ca}^{2+}$ oscillations [19]. Oscillations are driven by fluctuations in channel opening. The stochasticity of intracellular $\mathrm{Ca}^{2+}$ dynamics is caused by the stochastic binding and unbinding of $\mathrm{IP}_{3}$ and $\mathrm{Ca}^{2+}$ to the small number of receptor molecules. Fluctuations cause spontaneous release in a single cluster. That leads to a release spike like the initial spikes in figures 4 and 7 . Such a large amplitude event can lead to the opening of neighboring clusters and finally, via a nucleation process, to a wave traveling through the whole cell. If that occurs repeatedly, oscillation-like processes follow [13]. Thus, the amplitude of the initial spike is responsible for the amplitude of the oscillations. Nucleation may occur at different spots in the cell essentially at the same time when the $\mathrm{IP}_{3}$ concentration is high [13], leading to almost regular periods.

Oscillations might as well be introduced by additional feedback, e.g. a $\mathrm{Ca}^{2+}$ feedback on $\mathrm{IP}_{3}$ production or the filling state of the endoplasmic reticulum. Our findings suggest that the initiation of global $\mathrm{Ca}^{2+}$ release would still occur by wave nucleation, since the $\mathrm{Ca}^{2+}$ dynamics would not undergo a local instability. The additional feedbacks would just modulate the nucleation probability periodically. 
The present study sheds new light on the interplay between localization and fluctuations. This feature does not hold exclusively for $\mathrm{IP}_{3}$ mediated $\mathrm{Ca}^{2+}$ liberation, but other intracellular pathways should exhibit it, too. The involvement of a small number of proteins in cell signaling has already been established, see [27] for instance. It will be interesting to apply the current method to networks of strongly localized reaction sites. That will expand our knowledge on fluctuation induced phenomena, which have been mostly studied by stochastic simulations. In addition, our results reveal that the deterministic limit still has surprises in store, as the period doubling (see figure 7) shows. Whether this sequence leads to chaos, is the subject of ongoing investigations.

\section{Acknowledgment}

This work was supported by the DFG, SFB 555, TP B9.

\section{Appendix A: Linearization}

Linearizing the dynamics of $m$ diffusing species and $n$ gating variables for $r<\bar{a}$ results in $\dot{x}=M x$ with

$$
x=\left(\begin{array}{l}
y \\
z
\end{array}\right), \quad M=\left(\begin{array}{cc}
A_{1} & 0 \\
B & A_{2}
\end{array}\right) .
$$

$A_{1}$ is an $m$ dimensional quadratic matrix and $B \in \mathbb{R}^{n \times m}$. The quadratic matrix $A_{2}$ has the dimension $\operatorname{dim} A_{2}=n$. It originates from the gating dynamics. Since they are described by rate equations, which are derived form master equations, the Frobenius Perron theorem assures that $A_{2}$ can be diagonalized. Additionally, all eigenvalues $\lambda_{i}$ of $A_{2}$ are real with $\max \lambda_{i}=0$. The structure of $M$ then entails that the eigenvalues of $M$ include those of $A_{2}$. The eigenvector corresponding to $\lambda_{i}$ is of the form $\left(0, \ldots, 0, q_{i}\right)^{t}$ with $\operatorname{dim} q_{i}=n$. Therefore, the general solution for $y$ does not depend on $\exp \left(\lambda_{i} t\right), i=1, \ldots, n$.

\section{Appendix B: Stationary states}

We here provide the analytical expressions for the stationary points of the gating variables. In the DK model, the eight states are given by

$$
\begin{array}{ll}
\bar{p}_{000}=d_{1} d_{2} d_{5} \gamma_{1}, & \bar{p}_{100}=d_{2} d_{5} I \gamma_{1}, \\
\bar{p}_{010}=d_{1} d_{2} \bar{c} \gamma_{1}, & \bar{p}_{001}=d_{3} d_{5} \bar{c} \gamma_{1}, \\
\bar{p}_{011}=d_{3} \bar{c}^{2} \gamma_{1}, & \bar{p}_{101}=d_{5} \bar{c} I \gamma_{1}, \\
\bar{p}_{110}=d_{2} \bar{c} I \gamma_{1}, & \bar{p}_{111}=\bar{c}^{2} I \gamma_{1},
\end{array}
$$

with $\gamma_{1}^{-1}=\left(\bar{c}+d_{5}\right)\left(d_{1} d_{2}+\bar{c} d_{3}+\bar{c} I+d_{2} I\right)$. De Young and Keizer assume that binding and unbinding happens more quickly for $\mathrm{IP}_{3}$ than for $\mathrm{Ca}^{2+}$. We therefore approximate 
the $\mathrm{IP}_{3}$ dynamics by their equilibria i.e. $p_{1 i j}=\beta_{i j} p_{0 i j}$. Thus, we reduce the DK model to four lumped states $p_{i j}:=p_{0 i j}+p_{1 i j}[12]$. The corresponding stationary points read

$$
\begin{array}{ll}
\bar{p}_{00}=d_{5} d_{6} \gamma_{2}, & \bar{p}_{01}=d_{5} \bar{c} \gamma_{2}, \\
\bar{p}_{10}=d_{6} \bar{c} \gamma_{2}, & \bar{p}_{11}=\bar{c}^{2} \gamma_{2},
\end{array}
$$

with $\gamma_{2}^{-1}=\left(\bar{c}+d_{5}\right)\left(\bar{c}+d_{6}\right)$ and $d_{6}=d_{2}\left(I+d_{1}\right) /\left(I+d_{3}\right)$. Evaluating the $\beta_{i j}$ and using the lumped state definition directly entails that equations (25) lead to the same stationary values $\bar{p}_{i j k}$ as the DK model e.g. $\bar{p}_{110}=I /\left(I+d_{5}\right) \bar{p}_{10}$.

A widely used simplification of the DK model is the Li Rinzel (LR) model [10]. Therefore, we derive the function $\kappa(\omega)$ (equation (19)) for this model, too. The LR model uses a time scale separation between $\mathrm{Ca}^{2+}$ activation and $\mathrm{Ca}^{2+}$ inhibition. Experiments show that the inhibitory processes are much slower than activation. Thus, it is possible to eliminate $\mathrm{Ca}^{2+}$ activation by using the corresponding equilibrium value. It results in a single gating variable $p_{h}$, which denotes the fraction of states that are not yet inhibited. Its stationary value is

$$
\bar{p}_{h}=\frac{d_{6}}{d_{6}+\bar{c}} .
$$

As for the lumped states, the stationary values $\bar{p}_{i k j}$ calculated from the LR model equal those of De Young and Keizer. For instance, we arrive at

$$
\bar{p}_{110}^{L R}=\frac{I}{I+d_{1}} \frac{\bar{c}}{\bar{c}+d_{5}} \bar{p}_{h}=\bar{p}_{110}^{D K}
$$

for the fraction of open subunits. This identity is directly reflected in the calculation of $\kappa(\omega)$ for the LR model. It can be cast into the form

$$
\kappa(\omega)=\left[a_{0} \frac{4\left(1-\bar{p}_{110}\right)}{\sqrt[3]{\left(4-3 \bar{p}_{110}\right)^{2}}} \frac{\bar{p}_{110}}{\bar{c}}\left\{\frac{d_{5}}{d_{5}+\bar{c}}-\frac{\bar{c}}{\frac{\omega}{a_{2}}+d_{6}+\bar{c}}\right\}\right]_{\bar{a}}
$$

that is very similar to equation (19).

\section{Appendix C: Numerical methods}

The geometry of the $\mathrm{IP}_{3} \mathrm{R}$ cluster imposes considerations on the discretization. As stated above, the radius of the active area measures only tens of nanometers, but the outer boundary is 5-100 $\mu \mathrm{m}$ away. A constant grid size that sufficiently resolves the dynamics in the cluster would lead to an enormous calculational effort. To reduce computation time, we use a grid with non uniform spacing. The mesh size is sufficiently small for $r \leq a_{0}$ and saturates at a larger value in the bulk. It entails that the usual discretization of the radial Laplacian

$$
\nabla_{r}^{2}=\frac{1}{r^{2}} \frac{\partial}{\partial r}\left(r^{2} \frac{\partial}{\partial r}\right)=\frac{\partial^{2}}{\partial r^{2}}+\frac{2}{r} \frac{\partial}{\partial r}
$$


cannot be applied. Let $\left\{r_{i}\right\}$ denote the set of grid points, $d r_{i}:=r_{i}-r_{i-1}$ the spacing, and $u_{i}$ an approximation to the concentration profile. Then a second order scheme for equation (29) reads

$$
L\left(u_{i}\right)=\frac{1}{r_{i}^{2}}\left\{\left(r_{i}+\frac{d r_{i+1}}{2}\right)^{2} \frac{u_{i+1}-u_{i}}{d r_{i+1}}-\left(r_{i}-\frac{d r_{i}}{2}\right)^{2} \frac{u_{i}-u_{i-1}}{d r_{i}}\right\} \frac{2}{d r_{i+1}+d r_{i}} .
$$

Moreover, we adopt a first order scheme for the time integration and $50 \%$ of the stability criterion [28].

\section{Glossary}

Fixed element Any reactive substance that is restricted to small spatial regions and that possesses two distinct states: activated and deactivated. A fixed element participates only in a reaction when it is activated.

Bistability Existence of two linearly stable solutions of a nonlinear system.

Cytosol The fluid portion of the cell in which all other internal compartments, e.g. the endoplasmic reticulum, are embedded.

Endoplasmic reticulum An extensive membranous network within the cell, which serves as a major intracellular $\mathrm{Ca}^{2+}$ store.

Hopf bifurcation Emergence or disappearance of an oscillatory solution upon variation of a parameter in a nonlinear system.

Linear stability Property of a stationary solution of a nonlinear system. The system relaxes back to this stationary state upon any infinitesimal perturbation from this state, if it is linearly stable, and amplifies any infinitesimal perturbation, if it is linearly unstable.

Receptor A specialized protein on a cell's membrane that binds to substances that effect the activities of the cell.

Saddle node bifurcation Emergence or disappearance of two stationary states upon variation of a parameter in a nonlinear system.

Second messenger A chemical signal that relays a hormonal message from a cell's surface to its interior.

[1] Alberts B, Bray D, Lewis J, Raff M, Roberts K and Watson J 1994 Molecular biology of the cell (New York: Garland Publishing)

[2] Berridge M, Lipp P and Bootmann M 2000 Nature Rev. Mol. Cell Biol. 1(1) 11-21

[3] Miyazaki S, Shirakawa H, Nakada K and Honda Y 1993 Dev. Biol. 158 62-78

[4] Szalai G, Krishnamurthy R and Hajnczky G 1999 EMBO J. 18 6349-6361

[5] Bers D M 2002 Nature 415 198-205

[6] Marchant J and Parker I 2001 EMBO J. 20 65-76

[7] Jiang Q X, Thrower E C, Chester D W, Ehrlich B E and Sigworth F J 2002 EMBO J. 21 3575-3581,

[8] Thul R and Falcke M 2004 Biophys. J. 86 2660-2673

[9] De Young G and Keizer J 1992 Proc. Natl. Acad. Sci. USA 89 9895-9899

[10] Li Y and Rinzel J 1994 J. theor. Biol. 166 461-473 
[11] Bär M, Falcke M, Tsimring L and Levine H 2000 Phys. Rev. Lett. 84 5664-5667

[12] Falcke M, Tsimring L and Levine H 2000 Phys. Rev. E 62 2636-2643

[13] Falcke M 2003 Biophys. J. 84 42-56

[14] Jung P and Shuai J 2001 Europhys. Lett. 56(1) 29-35

[15] Shuai P and Jung P 2002 Biophys. J. 83(1) 87-97

[16] Falcke M 2004 Advances in Physics 53 255-440

[17] Swillens S, Dupont G, Combettes L and Champeil P 1999 Proc. Natl. Acad. Sci. USA 96 1375013755

[18] Bezprozvanny I, Watras J and Ehrlich B 1991 Nature 351 751-754

[19] Thul R and Falcke M 2004 Phys. Rev. Lett. 93 188103-1-4

[20] Adkins C and Taylor C 1999 Curr. Biol. 9 1115-1118

[21] Mak D, McBride S and Foskett J 2003 J. Gen. Physiol. 122 583-603

[22] Yao Y, Choi J and Parker I 1996 J. Physiol. 482 533-554

[23] Mak D, McBride S and Foskett J 1998 Proc. Natl. Acad. Sci. USA 95 15821-15825

[24] Mak D, McBride S and Foskett J 2001 J. Gen. Physiol. 117 435-446

[25] Ramos-Franco J, Fill M and Mignery G 1998 Biophys. J. 75 834-839

[26] Keener J and Sneyd J 1998 Mathematical physiology (New York: Springer)

[27] Howard M and Rutenberg A 2003 Phys. Rev. Lett. 90 128102-1-4

[28] Press W, Teukolsky S, Vetterling W and Flannery B 2002 Numerical recipes in $C++$ 2nd ed (Cambridge: Cambridge University Press) 